\title{
Plasma effect in tape helix traveling-wave tube
}

\author{
S. Saviz $\cdot$ F. Salehizadeh
}

Received: 8 March 2014/ Accepted: 21 April 2014/Published online: 21 May 2014

(C) The Author(s) 2014. This article is published with open access at Springerlink.com

\begin{abstract}
A linearized relativistic field theory of a plasma-loaded helix traveling-wave tube is presented for a configuration where a solid electron beam propagate through a sheath helix enclosed within a loss-free wall in which the gap between the helix and the outer wall is filled with a dielectric. Numerical study of the effect of plasma density on the phase velocity and growth rate has been done. Numerical results show that the plasma have different behaviors in different density limits.
\end{abstract}

Keywords Traveling wave tube $\cdot$ Plasma $\cdot$ Helix

\section{Introduction}

The relativistic traveling-wave tube (TWT) is an important high-power source of broad-band high-power microwave generation at centimeter wavelengths, developed over the last several decades [1-5]. One of the common features of a TWT is a slow-wave structure (SWS) such as a dielectric material, disk-loaded waveguide, or a helix [6-10]. The physical mechanism of operation is that the SWS reduces the phase velocity of the electromagnetic wave to synchronize it with the electron beam velocity, so that a strong interaction between the two can take place. This phenomenon of Cerenkov emission is the basis for all TWTs.

Pierce and his coworkers [11-13] employed the coupledwave analysis in their pioneering work, and the analysis of TWT improved using linear theories based on the Maxwell's equations in a sheath helix $[14,15]$. The coupledwave Pierce theory recovers the near-resonant limit. Both

S. Saviz $(\square) \cdot$ F. Salehizadeh

Plasma Physics Research Center, Science and Research Branch, Islamic Azad University, Tehran, Iran

e-mail: azarabadegan@gmail.com coupled-wave and field theories of TWT have discussed in [16] and [17]. Freund and coworkers developed the field theories of beam-loaded helix TWTs for tape helix model [18]. Freund and coworkers [19] described the numerical comparison between the complete dispersion equation and the Pierce model in helix TWT and shown that the coupledwave theory breaks down for sufficiently high currents. The complete field theory is more exact than the coupled-wave theory. A common feature of all these theories is that they ignore the effects of any background plasma.

However, the residual gases in the container get ionized by the beam and plasma of sensible density is formed. It has been found experimentally that injection of plasma into the microwave devices may enhance the interaction and the output power. Plasma filling can also improve the transmission quality of electron beam, even make beam transmission without a guiding magnetic field [20, 21].

The specific geometry under study is illustrated in Fig. 1. A conducting waveguide of radius $R_{\mathrm{g}}$ encloses a hollow dielectric with an inner radius $R_{\mathrm{h}}=R_{\mathrm{d}}$. Background plasma with a uniform density distribution of radius $R_{\mathrm{p}}$ is loaded inside the helix. The electron beam with radius $R_{\mathrm{b}}<R_{\mathrm{p}}$ propagates through the plasma.

The purpose is to investigate the effects of dielectric constant, plasma density, axial guide magnetic field, and electron beam density on the phase velocity and growth rate. In this manuscript, we expand a complete self-consistent and relativistic field theory of the plasma-loaded helix TWT by solution of the relativistic fluid equation and Maxwell's equations. The boundary condition includes three regions: (1) inside the beam that includes the electron beam and plasma, (2) between the beam and helix that includes the plasma, (3) between the helix and the wall that is dielectric.

The organization of the paper is as follows: "The model description" is determined in Sect. 2 through application of 


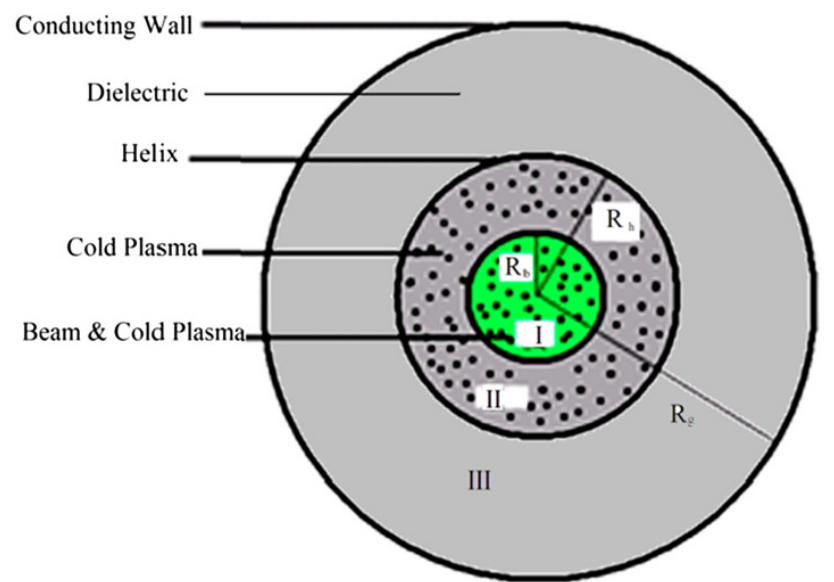

Fig. 1 Cross-sectional view of the structure. The dielectric fills the region between $R_{\mathrm{h}}$ (helix radius) and $R_{\mathrm{g}}$ (conducting wall radius) and plasma fills the region between 0 and $R_{\mathrm{h}}$. The relativistic electron beam along with the cold plasma fills the region between $R=0$ and $R_{\mathrm{b}}$

the appropriate boundary conditions upon the solution of Maxwell's equations. In Sect. 3, we deal with "Numerical results and discussions" and finally some of the "Conclusions" are given in 4 .

\section{The model description}

With the aid of the Maxwell and fluid equations the wave equations in regions I and II are given by Region I

$$
\begin{aligned}
& \left(\nabla_{\perp}^{2}+\chi_{ \pm, \mathrm{I}, \mathrm{bp}}^{2}\right) \delta \hat{E}_{z}=0 \\
& \left(\nabla_{\perp}^{2}+\chi_{ \pm, \mathrm{I}, \mathrm{bp}}^{2}\right) \delta \hat{B}_{z}=0
\end{aligned}
$$

Region II

$$
\begin{aligned}
\left(\nabla_{\perp}^{2}+\right. & \left.\chi_{ \pm, \mathrm{II}, \mathrm{p}}^{2}\right) \delta \hat{E}_{z}=0 \\
\left(\nabla_{\perp}^{2}+\right. & \left.\chi_{ \pm, \mathrm{II}, \mathrm{p}}^{2}\right) \delta \hat{B}_{z}=0 \\
\chi_{ \pm, \mathrm{I}, \mathrm{bp}} & =\left(2\left[1-\frac{\omega_{\mathrm{b}}^{2}}{\gamma_{0 \mathrm{e}}^{2}\left(\Delta \omega^{2}-\Omega_{\mathrm{ce}}^{2}\right)}+\Lambda_{+\mathrm{bp}} \Lambda_{-\mathrm{bp}} Y 3\right]\right)^{-1} \\
& \times\left\{\left[1-\frac{\omega_{\mathrm{b}}^{2}}{\gamma_{0 \mathrm{e}}^{2}\left(\Delta \omega^{2}-\Omega_{\mathrm{ce}}^{2}\right)}+\Lambda_{s c}\left(1-2 \alpha_{\mathrm{b}}\right)\right] \chi^{2}+\Lambda_{+\mathrm{bp}} \Lambda_{-\mathrm{bp}} Y 1\right\} \\
& \mp\left\{1-\frac{\omega_{\mathrm{b}}^{2}}{\gamma_{\mathrm{0e}}^{2}\left(\Delta \omega^{2}-\Omega_{\mathrm{ce}}^{2}\right)}+\Lambda_{\mathrm{sc}}\left(1-2 \alpha_{\mathrm{b}}\right)\right]^{2} \chi^{4} \\
& +\left(\Lambda_{+\mathrm{bp}} \Lambda_{-\mathrm{bp}} Y 1\right)^{2}+2 \Lambda_{+\mathrm{bp}} \Lambda_{-\mathrm{bp}} Y 1 \chi^{2} \\
& \times\left[1-\frac{\omega_{\mathrm{b}}^{2}}{\lambda_{0 \mathrm{e}}^{2}\left(\Delta \omega^{2}-\Omega_{\mathrm{ce}}^{2}\right)}+\Lambda_{\mathrm{sc}}\left(1-2 \alpha_{\mathrm{b}}\right)\right] \\
& \left.-4 \Lambda_{\mathrm{sc}} \Lambda_{+\mathrm{bp}} \Lambda_{-\mathrm{bp}} \chi^{4}\left(1-\frac{\omega_{\mathrm{b}}^{2}}{\gamma_{0 \mathrm{e}}^{2}\left(\Delta \omega^{2}-\Omega_{\mathrm{ce}}^{2}\right)}\right)-4 Y 4\left(\Lambda_{+\mathrm{bp}} \Lambda_{-\mathrm{bp}}\right)^{2}\right\}^{1 / 2}
\end{aligned}
$$

$$
\begin{aligned}
\chi_{ \pm, \mathrm{II}, \mathrm{p}}= & \chi^{2}\left(2\left[1-\frac{\omega_{\mathrm{p}}^{2} \gamma_{0 \mathrm{e}}}{\left(\omega^{2}-\left(\gamma_{0 \mathrm{e}} \Omega_{\mathrm{ce}}\right)^{2}\right)}\right]\right)^{-1} \\
& \times\left\{\left[1-\frac{\omega_{\mathrm{p}}^{2} \gamma_{0 \mathrm{e}}}{\left(\omega^{2}-\left(\gamma_{0 \mathrm{e}} \Omega_{\mathrm{ce}}\right)^{2}\right)}+\Lambda_{\mathrm{scp}}\left(1-2 \alpha_{\mathrm{p}}\right)\right]^{2}\right. \\
& \mp\left\{1-\frac{\omega_{\mathrm{p}}^{2} \gamma_{0 \mathrm{e}}}{\left(\omega^{2}-\left(\gamma_{0 \mathrm{e}} \Omega_{\mathrm{ce}}\right)^{2}\right)}+\Lambda_{\mathrm{scp}}\left(1-2 \alpha_{\mathrm{p}}\right)\right]^{2} \\
& \left.\left.-4 \Lambda_{\mathrm{scp}} \Lambda_{+\mathrm{p}} \Lambda_{-\mathrm{p}}\left(1-\frac{\omega_{\mathrm{p}}^{2} \gamma_{0 \mathrm{e}}}{\left(\omega^{2}-\left(\gamma_{0 \mathrm{e}} \Omega_{\mathrm{ce}}\right)^{2}\right)}\right)\right\}\right\}^{1 / 2} \\
\Lambda_{\mathrm{sc}}= & 1-\frac{\omega_{\mathrm{b}}^{2}}{\Delta \omega^{2} \gamma_{0 \mathrm{e}}^{2}}, \Lambda_{\mathrm{scp}}=1-\frac{\omega_{\mathrm{p}}^{2} \gamma_{0 \mathrm{e}}}{\omega^{2}}, \\
Y_{1}= & \frac{\chi^{2} \Lambda_{\mathrm{sc}}\left(B_{\mathrm{p}}+B_{\mathrm{bp}}\right)}{\Lambda_{+\mathrm{bp}} \Lambda_{-\mathrm{bp}}}-\left(1+\frac{\alpha_{\mathrm{b}} \Lambda_{0 \mathrm{~b}}}{\Lambda_{+\mathrm{bp}} \Lambda_{-\mathrm{bp}}}\right)\left(\frac{\chi^{2} \omega_{\mathrm{p}}^{2} \gamma_{0 \mathrm{e}}}{\omega^{2}}\right) \\
+ & \left(\frac{\chi^{2} \omega_{\mathrm{p}}^{2} \gamma_{0 \mathrm{e}}}{\omega^{2}}\right)\left(\frac{\left(B_{\mathrm{p}}+B_{\mathrm{bp}}\right)}{\Lambda_{+\mathrm{bp}} \Lambda_{-\mathrm{bp}}}\right)+\frac{\left(c k-\omega \beta_{0 \mathrm{e}}\right) \chi^{2} \alpha_{\mathrm{b}}}{\Delta \omega^{2}} \\
& \times\left\{\frac{\Delta \omega}{\Lambda_{+b p} \Lambda_{-b p}}\left(A_{\mathrm{p}}+A_{\mathrm{bp}}\right)-\frac{i \Omega_{\mathrm{ce}}}{\Lambda_{+\mathrm{bp}} \Lambda_{-\mathrm{bp}}}\left(D_{\mathrm{p}}+D_{\mathrm{bp}}\right)\right\} \\
+ & \frac{\chi^{2} \alpha_{\mathrm{p}}(k c)}{\omega^{2}} \bar{A}_{\mathrm{p}, I, 1}
\end{aligned}
$$

$$
\begin{aligned}
Y_{3}= & -\left\{1+\Delta \omega^{-2} \alpha_{\mathrm{b}}\left(c k-\omega \beta_{0 \mathrm{e}}\right)^{2} .\right. \\
& \left.+\left[1+\frac{\alpha_{\mathrm{b}}}{\Lambda_{+\mathrm{bp}} \Lambda_{-\mathrm{bp}}}\left(\Lambda_{0 \mathrm{~b}}+\frac{\Omega_{\mathrm{ce}}^{2}}{\Delta \omega^{2}}\right)\right]\right\}\left(\frac{B_{\mathrm{p}}+B_{\mathrm{bp}}}{\Lambda_{+\mathrm{bp}} \Lambda_{-\mathrm{bp}}}\right) \\
& +\left(1+\frac{\alpha_{\mathrm{b}} \Lambda_{0 \mathrm{~b}}}{\Lambda_{+\mathrm{bp}} \Lambda_{-\mathrm{bp}}}\right)\left\{\frac { ( c k - \omega \beta _ { 0 \mathrm { e } } ) \alpha _ { \mathrm { b } } } { \Delta \omega ^ { 2 } } \left\{\frac{\Delta \omega}{\Lambda_{+\mathrm{bp}} \Lambda_{-\mathrm{bp}}}\left(A_{\mathrm{p}}+A_{\mathrm{bp}}\right)\right.\right. \\
& \left.\left.-\frac{i \Omega_{\mathrm{ce}}}{\Lambda_{+\mathrm{pp}} \Lambda_{-\mathrm{bp}}}\left(D_{\mathrm{p}}+D_{\mathrm{bp}}\right)\right\}+\frac{\alpha_{\mathrm{p}}(k c)}{\omega^{2}} \bar{A}_{\mathrm{p}, I, 1}\right\} \\
& +\left(\frac{B_{\mathrm{p}}+B_{\mathrm{bp}}}{\Lambda_{+\mathrm{bp}} \Lambda_{-\mathrm{bp}}}\right)\left\{\frac { ( c k - \omega \beta _ { 0 \mathrm { e } } ) \alpha _ { \mathrm { b } } } { \Delta \omega ^ { 2 } } \left\{\frac{\Delta \omega}{\Lambda_{+\mathrm{bp}} \Lambda_{-\mathrm{bp}}}\left(A_{\mathrm{p}}+A_{\mathrm{bp}}\right)\right.\right. \\
E_{\mathrm{p}}- & \left.\frac{i \Omega_{\mathrm{ce}}}{\Lambda_{+\mathrm{bp}} \Lambda_{-\mathrm{bp}}}\left(B_{\mathrm{p}}+B_{\mathrm{bp}}\right)\right]+\frac{i \alpha_{\mathrm{b}} \Omega_{\mathrm{ce}}\left(c k-\omega \beta_{0 \mathrm{e}}\right)}{\Delta \omega^{2}} \\
& \times\left[1+\frac{\alpha_{\mathrm{b}}}{\Lambda_{+\mathrm{bp}} \Lambda_{-\mathrm{bp}}}\left(1+\Lambda_{0 \mathrm{~b}}\right)\right]\left[\frac{D_{\mathrm{p}}+D_{\mathrm{bp}}}{\Lambda_{+\mathrm{bp}} \Lambda_{-\mathrm{bp}}}\right] \\
& +\left\{\left[\frac{\alpha_{\mathrm{b}}\left(c k-\omega \beta_{0 \mathrm{e}}\right)}{\Delta \omega^{2}}\right]\left[\frac{\Delta \omega}{\Lambda_{+\mathrm{bp}} \Lambda_{-\mathrm{bp}}} E_{\mathrm{p}}-\frac{i \Omega_{\mathrm{ce}}}{\Lambda_{+\mathrm{bp}} \Lambda_{-\mathrm{bp}}}\left(B_{\mathrm{p}}+B_{\mathrm{bp}}\right)\right]\right. \\
& \left.+\frac{\alpha_{\mathrm{p}}(k c)}{\omega^{2}} \bar{A}_{\mathrm{p}, I, 2}\right\}\left\{\frac{i \alpha_{\mathrm{b}} \Omega_{\mathrm{ce}}}{\Lambda_{+\mathrm{bp}} \Lambda_{-\mathrm{bp}}} \frac{\left(c k-\omega \beta_{0 \mathrm{e}}\right)}{\Delta \omega^{2}}\right\} \\
& +\left[\frac{D_{\mathrm{p}}+D_{b p}}{\Lambda_{+\mathrm{bp}} \Lambda_{-\mathrm{bp}}}\right]\left\{\left[\frac{\alpha_{\mathrm{b}}\left(c k-\omega \beta_{0 \mathrm{e}}\right)}{\Delta \omega^{2}}\right]\right. \\
& \left.\times\left[\frac{\Delta \omega}{\Lambda_{+b p} \Lambda_{-b p}} E_{p}-\frac{i \Omega_{\mathrm{ce}}}{\Lambda_{+\mathrm{bp}} \Lambda_{-\mathrm{bp}}}\left(B_{\mathrm{p}}+B_{\mathrm{bp}}\right)\right]+\frac{\alpha_{\mathrm{p}}(k c)}{\omega^{2}} \bar{A}_{\mathrm{p}, I, 2}\right\}
\end{aligned}
$$




$$
\begin{aligned}
& Y_{4}=Y_{3}\left[\chi^{4} \Lambda_{\mathrm{sc}}-\chi^{4} \frac{\omega_{\mathrm{p}}^{2} \gamma_{0 \mathrm{e}}}{\omega^{2}}\right]-\chi^{4} \frac{\omega_{\mathrm{p}}^{2} \gamma_{0 \mathrm{e}}}{\omega^{2}}\left[\left(1+\frac{\alpha_{\mathrm{b}} \Lambda_{0 \mathrm{~b}}}{\Lambda_{\mathrm{bp}} \Lambda_{-\mathrm{bp}}}\right)\right. \\
& \times\left\{1+\alpha_{\mathrm{b}}\left(c k-\omega \beta_{0 \mathrm{e}}\right)^{2}\left[1+\frac{\alpha_{\mathrm{b}}}{\Lambda_{+\mathrm{bp}} \Lambda_{-\mathrm{bp}}}\left(\Lambda_{0 \mathrm{~b}}+\frac{\Omega_{\mathrm{ce}}^{2}}{\Delta \omega^{2}}\right)\right] / \Delta \omega^{2}\right\} \\
& \left.-\frac{\alpha_{\mathrm{b}}^{2} \Omega_{\mathrm{ce}}^{2}\left(c k-\omega \beta_{0 \mathrm{e}}\right)^{2}}{\Delta \omega^{4} \Lambda_{+\mathrm{bp}} \Lambda_{-\mathrm{bp}}}\left\{1+\frac{\alpha_{\mathrm{b}}}{\Lambda_{+\mathrm{bp}} \Lambda_{-\mathrm{bp}}}\left(1+\Lambda_{0 \mathrm{~b}}\right)\right\}\right] \\
& \bar{B}_{\mathrm{p}, I I, 1}=c k\left[1+\frac{\alpha_{\mathrm{p}}}{\Lambda_{+\mathrm{p}} \Lambda_{-\mathrm{p}}}\left[\Lambda_{0 \mathrm{p}}+\frac{\left(\Omega_{\mathrm{ce}} \gamma_{0 \mathrm{e}}\right)^{2}}{\omega^{2}}\right]\right], \\
& B_{\mathrm{p}, I I, 2}=\Omega_{\mathrm{ce}} \gamma_{0 \mathrm{e}}\left[1+\frac{\alpha_{\mathrm{p}}}{\Lambda_{+\mathrm{p}} \Lambda_{-\mathrm{p}}}\left[1+\Lambda_{0 \mathrm{p}}\right]\right] . \\
& \bar{A}_{\mathrm{e}, \mathrm{I}, 1}=\left(k c-\omega \beta_{0 \mathrm{e}}\right)\left[1+\frac{\alpha_{\mathrm{b}}}{\Lambda_{+\mathrm{bp}} \Lambda_{-\mathrm{bp}}}\left(\Lambda_{0 \mathrm{~b}}+\frac{\Omega_{\mathrm{ce}}^{2}}{\Delta \omega^{2}}\right)\right] \\
& +\frac{\Delta \omega}{\Lambda_{+\mathrm{bp}} \Lambda_{-\mathrm{bp}}}\left[\left(A_{\mathrm{p}}+A_{\mathrm{bp}}\right]-\frac{i \Omega_{\mathrm{ce}}}{\Lambda_{+\mathrm{bp}} \Lambda_{-\mathrm{bp}}}\left[\left(D_{\mathrm{p}}+D_{\mathrm{bp}}\right]\right.\right. \\
& \bar{A}_{\mathrm{e}, \mathrm{I}, 2}=i \Omega_{\mathrm{ce}}\left[1+\frac{\alpha_{\mathrm{b}}}{\Lambda_{+\mathrm{bp}} \Lambda_{-\mathrm{bp}}}\left(1+\Lambda_{0 \mathrm{~b}}\right)\right] \\
& +\frac{\Delta \omega}{\Lambda_{+\mathrm{bp}} \Lambda_{-\mathrm{bp}}} E_{\mathrm{p}}-\frac{i \Omega_{\mathrm{ce}}}{\Lambda_{+\mathrm{bp}} \Lambda_{-\mathrm{bp}}}\left[B_{\mathrm{p}}+B_{\mathrm{bp}}\right] \\
& \left.\bar{A}_{\mathrm{p}, \mathrm{I}, 1}=k c+\frac{\alpha_{\mathrm{b}}\left(k c-\omega \gamma_{0 \mathrm{e}}\right)}{\Lambda_{+\mathrm{bp}} \Lambda_{-\mathrm{bp}}}\left[\frac{\omega}{\Delta \omega} \Lambda_{0 \mathrm{~b}}+\frac{\Omega_{\mathrm{ce}}^{2} \gamma_{0 e}}{\Delta \omega^{2}}\right)\right] \\
& +\frac{\omega}{\Lambda_{+\mathrm{bp}} \Lambda_{-\mathrm{bp}}}\left[\left(A_{\mathrm{p}}+A_{\mathrm{bp}}\right]-\frac{i \Omega_{\mathrm{ce}} \gamma_{0 \mathrm{e}}}{\Lambda_{+\mathrm{bp}} \Lambda_{-\mathrm{bp}}}\left[\left(D_{\mathrm{p}}+D_{\mathrm{bp}}\right]\right.\right. \\
& \bar{A}_{\mathrm{p}, \mathrm{I}, 2}=i \Omega_{\mathrm{ce}} \gamma_{0 \mathrm{e}}\left\{1+\frac{\alpha_{\mathrm{b}}}{\Lambda_{\mathrm{bp}} \Lambda_{-\mathrm{bp}}}\left[\frac{\omega}{\Delta \omega \gamma_{0 \mathrm{e}}}+\Lambda_{0 \mathrm{~b}}\right]\right\} \\
& +\frac{\omega}{\Lambda_{+\mathrm{bp}} \Lambda_{-\mathrm{bp}}} E_{p}-\frac{i \Omega_{\mathrm{ce}} \gamma_{0 \mathrm{e}}}{\Lambda_{+\mathrm{bp}} \Lambda_{-\mathrm{bp}}}\left[B_{\mathrm{p}}+B_{\mathrm{bp}}\right]
\end{aligned}
$$

Having applied the boundary condition at the waveguide wall and edge of the beam, we obtain

$$
\delta \hat{E}_{z}=\left\{\begin{array}{l}
A J_{0}\left(\chi_{ \pm, I \mathrm{bp}} r\right), \quad 0 \leq r \leq R_{\mathrm{b}} \\
A\left[a^{(1)} J_{0}\left(\chi_{ \pm, \mathrm{II}, \mathrm{p}} r\right)+a^{(2)} Y_{0}\left(\chi_{ \pm, \mathrm{II}, \mathrm{p}} r\right)\right] \\
\quad+i F\left[c^{(1)} J_{0}\left(\chi_{ \pm, \mathrm{II}, \mathrm{p}} r\right)+c^{(2)} Y_{0}\left(\chi_{ \pm, \mathrm{II}, \mathrm{p}} r\right)\right], \quad R_{\mathrm{b}}<r \leq R_{\mathrm{h}} \\
D W\left(q R_{\mathrm{g}}, q r\right), \quad R_{\mathrm{h}}<r \leq R_{\mathrm{g}}
\end{array}\right.
$$

$$
\delta \hat{B}_{z}=\left\{\begin{array}{l}
F J_{0}\left(\chi_{ \pm, I, \mathrm{bp}} r\right), \quad 0 \leq r \leq R_{\mathrm{b}} \\
F\left[b^{(1)} J_{0}\left(\chi_{ \pm, \mathrm{II}, \mathrm{p}} r\right)+b^{(2)} Y_{0}\left(\chi_{ \pm, \mathrm{II}, \mathrm{p}} r\right)\right] \\
\quad+i A\left[d^{(1)} J_{0}\left(\chi_{ \pm, \mathrm{II}, \mathrm{p}} r\right)+d^{(2)} Y_{0}\left(\chi_{ \pm, \mathrm{II}, \mathrm{p}} r\right)\right], \quad R_{\mathrm{b}}<r \leq R_{\mathrm{h}} \\
K W^{\prime}\left(q R_{\mathrm{g}}, q r\right), R_{\mathrm{h}}<r \leq R_{\mathrm{g}}
\end{array}\right.
$$

$$
\begin{aligned}
& q=\left(\varepsilon \omega^{2} / c^{2}-k^{2}\right)^{1 / 2} \\
& a^{(1)}=\frac{1}{G^{(2)}}\left[-J_{0}\left(\chi_{ \pm, \mathrm{I}, \mathrm{bp}} R_{\mathrm{b}}\right) \varsigma_{14}+Y_{0}\left(\chi_{ \pm, \mathrm{II}, \mathrm{p}} R_{\mathrm{b}}\right) \varsigma_{12}\right], \\
& a^{(2)}=\frac{1}{G^{(2)}}\left[J_{0}\left(\chi_{ \pm, \mathrm{I}, \mathrm{bp}} R_{\mathrm{b}}\right) \varsigma_{13}-J_{0}\left(\chi_{ \pm, \mathrm{II}, \mathrm{p}} R_{\mathrm{b}}\right) \varsigma_{12}\right], \\
& c^{(1)}=-\frac{1}{G^{(2)}}\left[Y_{0}\left(\chi_{ \pm, \mathrm{II}, \mathrm{p}} R_{\mathrm{b}}\right) \varsigma_{11}\right], c^{(2)}=\frac{1}{G^{(2)}}\left[J_{0}\left(\chi_{ \pm, \mathrm{II}, \mathrm{p}} R_{\mathrm{b}}\right) \varsigma_{11}\right], \\
& b^{(1)}=\frac{1}{G^{(1)}}\left[-J_{0}\left(\chi_{ \pm, \mathrm{I}, \mathrm{bp}} R_{\mathrm{b}}\right) \vartheta_{14}+Y_{0}\left(\chi_{ \pm, \mathrm{II}, \mathrm{p}} R_{\mathrm{b}}\right) \vartheta_{12}\right], \\
& b^{(2)}=\frac{1}{G^{(1)}}\left[-J_{0}\left(\chi_{ \pm, \mathrm{I}, \mathrm{bp}} R_{\mathrm{b}}\right) \vartheta_{13}-J_{0}\left(\chi_{ \pm, \mathrm{II}, \mathrm{p}} R_{\mathrm{b}}\right) \vartheta_{12}\right], \\
& d^{(2)}=-\frac{1}{G^{(1)}}\left[J_{0}\left(\chi_{ \pm, \mathrm{II}, \mathrm{p}} R_{\mathrm{b}}\right) \vartheta_{11}\right], \\
& G^{(1)}=\left[Y_{0}\left(\chi_{ \pm, \mathrm{II}, \mathrm{p}} R_{\mathrm{b}}\right) \vartheta_{13}-J_{0}\left(\chi_{ \pm, \mathrm{II}, \mathrm{p}} R_{\mathrm{b}}\right) \vartheta_{14}\right], \\
& d^{(1)}=\frac{1}{G^{(1)}}\left[Y_{0}\left(\chi_{ \pm, \mathrm{II}, \mathrm{p}} R_{\mathrm{b}}\right) \vartheta_{11}\right], \\
& G^{(2)}=\left[Y_{0}\left(\chi_{ \pm, \mathrm{II}, \mathrm{p}} R_{\mathrm{b}}\right) \vartheta_{13}-J_{0}\left(\chi_{ \pm, \mathrm{II}, \mathrm{p}} R_{b}\right) \vartheta_{14}\right], \\
& \vartheta_{11}=\frac{\chi_{ \pm, \mathrm{II}, \mathrm{p}}}{\chi_{ \pm, \mathrm{I}, \mathrm{bp}}}\left[\Theta 7 \frac{\chi_{ \pm, \mathrm{II}, \mathrm{p}}}{\chi_{ \pm, \mathrm{I}, \mathrm{bp}}} J_{0}\left(\chi_{ \pm, \mathrm{I}, \mathrm{bp}} R_{\mathrm{b}}\right)-S J_{0}\left(\chi_{ \pm, \mathrm{II}, \mathrm{p}} R_{\mathrm{b}}\right)\right] \\
& \times[\Theta 4 \Theta 7-\Theta 3 \Theta 8]-\frac{\chi_{ \pm, \mathrm{II}, \mathrm{p}}}{\chi_{ \pm, \mathrm{I}, \mathrm{bp}}} \\
& {\left[\Theta 7 Y_{0}\left(\chi_{ \pm, \mathrm{II}, \mathrm{p}} R_{\mathrm{b}}\right)-\Theta 8 J_{0}\left(\chi_{ \pm, \mathrm{II}, \mathrm{p}} R_{\mathrm{b}}\right)\right][P \Theta 7-S \Theta 3]} \\
& \vartheta_{12}=\frac{\chi_{ \pm, \mathrm{II}, \mathrm{p}}}{\chi_{ \pm, \mathrm{I}, \mathrm{bp}}}\left[T J_{0}\left(\chi_{ \pm, \mathrm{II}, \mathrm{p}} R_{\mathrm{b}}\right)\right][\Theta 4 \Theta 7-\Theta 3 \Theta 8] \\
& -[Q \Theta 7+T \Theta 3] \frac{\chi_{ \pm, \mathrm{II}, \mathrm{p}}}{\chi_{ \pm, \mathrm{I}, \mathrm{bp}}}\left[\Theta 7 Y_{0}\left(\chi_{ \pm, \mathrm{II}, \mathrm{p}} R_{\mathrm{b}}\right)-\Theta 8 J_{0}\left(\chi_{ \pm, \mathrm{II}, \mathrm{p}} R_{\mathrm{b}}\right)\right] \\
& \vartheta_{13}=\left(\frac{\chi_{ \pm, \mathrm{II}, \mathrm{p}}}{\chi_{ \pm, \mathrm{I}, \mathrm{bp}}}\right)^{2}[\Theta 4 \Theta 7-\Theta 3 \Theta 8]\left[\Theta 5 J_{0}\left(\chi_{ \pm, \mathrm{II}, \mathrm{p}} R_{b}\right)\right] \\
& -\left(\frac{\chi_{ \pm, \mathrm{II}, \mathrm{p}}}{\chi_{ \pm, \mathrm{I}, \mathrm{bp}}}\right)^{2}\left[\Theta 7 Y_{0}\left(\chi_{ \pm, \mathrm{II}, \mathrm{p}} R_{\mathrm{b}}\right)-\Theta 8 J_{0}\left(\chi_{ \pm, \mathrm{II}, \mathrm{p}} R_{\mathrm{b}}\right)\right] \\
& \times[\Theta 7 \Theta 1+\Theta 5 \Theta 3] \\
& \vartheta_{14}=\left(\frac{\chi_{ \pm, \mathrm{II}, \mathrm{p}}}{\chi_{ \pm, \mathrm{I}, \mathrm{bp}}}\right)^{2}\left[\Theta 6 J_{0}\left(\chi_{ \pm, \mathrm{II}, \mathrm{p}} R_{\mathrm{b}}\right)\right][\Theta 4 \Theta 7-\Theta 3 \Theta 8] \\
& -\left(\frac{\chi_{ \pm, \mathrm{II}, \mathrm{p}}}{\chi_{ \pm, \mathrm{I}, \mathrm{pp}}}\right)^{2}\left[\Theta 7 Y_{0}\left(\chi_{ \pm, \mathrm{II}, \mathrm{p}} R_{\mathrm{b}}\right)-\Theta 8 J_{0}\left(\chi_{ \pm, \mathrm{II}, \mathrm{p}} R_{\mathrm{b}}\right)\right] \\
& \times[\Theta 2 \Theta 7+\Theta 6 \Theta 3] \\
& \varsigma_{11}=\frac{\chi_{ \pm, \mathrm{II}, \mathrm{p}}}{\chi_{ \pm, \mathrm{I}, \mathrm{bp}}}\left[\Theta 5 \frac{\chi_{ \pm, \mathrm{II}, \mathrm{p}}}{\chi_{ \pm, \mathrm{I}, \mathrm{bp}}} J_{0}\left(\chi_{ \pm, \mathrm{I}, \mathrm{bp}} R_{\mathrm{b}}\right)-T J_{0}\left(\chi_{ \pm, \mathrm{II}, \mathrm{p}} R_{\mathrm{b}}\right)\right] \\
& \times[\Theta 2 \Theta 5-\Theta 1 \Theta 6]-\frac{\chi_{ \pm, \mathrm{II}, \mathrm{p}}}{\chi_{ \pm, \mathrm{I}, \mathrm{bp}}}\left[\Theta 5 Y_{0}\left(\chi_{ \pm, \mathrm{II}, \mathrm{p}} R_{\mathrm{b}}\right)\right. \\
& \left.-\Theta 6 J_{0}\left(\chi_{ \pm, \mathrm{II}, \mathrm{p}} R_{\mathrm{b}}\right)\right][Q \Theta 5-T \Theta 1]
\end{aligned}
$$




$$
\begin{aligned}
\varsigma_{12}= & \frac{\chi_{ \pm, \mathrm{II}, \mathrm{p}}}{\chi_{ \pm, \mathrm{I}, \mathrm{bp}}}\left[S J_{0}\left(\chi_{ \pm, \mathrm{II}, \mathrm{p}} R_{b}\right)\right][\Theta 2 \Theta 5-\Theta 1 \Theta 6] \\
& -[P \Theta 5+S \Theta 1] \frac{\chi_{ \pm, \mathrm{II}, \mathrm{p}}}{\chi_{ \pm, \mathrm{I}, \mathrm{bp}}}\left[\Theta 5 Y_{0}\left(\chi_{ \pm, \mathrm{II}, \mathrm{p}} R_{b}\right)-\Theta 6 J_{0}\left(\chi_{ \pm, \mathrm{II}, \mathrm{p}} R_{\mathrm{b}}\right)\right] \\
\varsigma_{13}= & \left(\frac{\chi_{ \pm, \mathrm{II}, \mathrm{p}}}{\chi_{ \pm, \mathrm{I}, \mathrm{bp}}}\right)^{2}[\Theta 2 \Theta 5-\Theta 1 \Theta 6]\left[\Theta 7 J_{0}\left(\chi_{ \pm, \mathrm{II}, \mathrm{p}} R_{\mathrm{b}}\right)\right] \\
& -\left(\frac{\chi_{ \pm, \mathrm{II}, \mathrm{p}}}{\chi_{ \pm, \mathrm{I}, \mathrm{bp}}}\right)^{2}\left[\Theta 5 Y_{0}\left(\chi_{ \pm, \mathrm{II}, \mathrm{p}} R_{\mathrm{b}}\right)-\Theta 6 J_{0}\left(\chi_{ \pm, \mathrm{II}, \mathrm{p}} R_{\mathrm{b}}\right)\right] \\
& \times[\Theta 3 \Theta 5+\Theta 7 \Theta 1] \\
\varsigma_{14}= & \left(\frac{\chi_{ \pm, \mathrm{II}, \mathrm{p}}}{\chi_{ \pm, \mathrm{I}, \mathrm{bp}}}\right)^{2}\left[\Theta 8 J_{0}\left(\chi_{ \pm, \mathrm{II}, \mathrm{p}} R_{\mathrm{b}}\right)\right][\Theta 2 \Theta 5-\Theta 1 \Theta 6] \\
& -\left(\frac{\chi_{ \pm, \mathrm{II}, \mathrm{p}}}{\chi_{ \pm, \mathrm{I}, \mathrm{bp}}}\right)^{2}\left[\Theta 5 Y_{0}\left(\chi_{ \pm, \mathrm{II}, \mathrm{p}} R_{\mathrm{b}}\right)-\Theta 6 J_{0}\left(\chi_{ \pm, \mathrm{II}, \mathrm{p}} R_{\mathrm{b}}\right)\right] \\
& \times[\Theta 4 \Theta 5+\Theta 1 \Theta 8]
\end{aligned}
$$

$W\left(q R_{\mathrm{g}}, q r\right)=Y_{0}\left(q R_{\mathrm{g}}\right) J_{0}(q r)-J_{0}\left(q R_{\mathrm{g}}\right) Y_{0}(q r)$,

$W^{\prime}\left(q R_{\mathrm{g}}, q r\right)=Y_{0}^{\prime}\left(q R_{\mathrm{g}}\right) J_{0}(q r)-J_{0}^{\prime}\left(q R_{\mathrm{g}}\right) Y_{0}(q r)$.

$$
\begin{aligned}
& P=\frac{1}{\Lambda_{+\mathrm{bp}} \Lambda_{-\mathrm{bp}}}\left\{\left[\alpha_{\mathrm{b}} \Lambda_{0 \mathrm{~b}} \frac{\left(c k-\omega \beta_{0 \mathrm{e}}\right)}{\Delta \omega}+\alpha_{\mathrm{p}} \Lambda_{0 \mathrm{p}} \frac{c k}{\omega}\right] J_{-1}\left(\chi_{ \pm, \mathrm{I}, \mathrm{bp}} R_{\mathrm{b}}\right)\right. \\
& -\alpha_{\mathrm{b}} \alpha_{\mathrm{p}}\left\{J _ { 0 } ^ { \prime } ( \chi _ { \pm , I , \mathrm { bp } } R _ { \mathrm { b } } ) \left\{( c k - \omega \beta _ { 0 \mathrm { e } } ) \left[\frac{1}{\Delta \omega^{2}}+\frac{\Omega_{c e} \beta_{0 \mathrm{e}}}{\omega \Delta \omega^{2}}\right.\right.\right. \\
& \left.\left.\left.\left.+\frac{\Omega_{\mathrm{ce}}}{\Delta \omega^{2}}-\frac{\Omega_{\mathrm{ce}} \beta_{0 \mathrm{e}}}{\omega \Delta \omega}\right]+c k\left[\frac{1}{\omega^{2}}+\frac{\Omega_{\mathrm{ce}}}{\Delta \omega \omega^{2}}+\frac{\Omega_{\mathrm{ce}} \beta_{0 e}}{\omega^{2}}-\frac{\Omega_{\mathrm{ce}}}{\omega \Delta \omega}\right]\right\}\right\}\right\} \\
& Q=\left\{-J_{0}^{\prime}\left(\chi_{ \pm, \mathrm{I}, \mathrm{bp}} R_{\mathrm{b}}\right)+\frac{1}{\Lambda_{+\mathrm{bp}} \Lambda_{-\mathrm{bp}}}\right. \\
& \times\left\{-\left[\alpha_{\mathrm{b}} \Lambda_{0 \mathrm{~b}}+\alpha_{\mathrm{p}} \Lambda_{0 \mathrm{p}}\right] J_{-1}\left(\chi_{ \pm, \mathrm{I}, \mathrm{bp}} R_{\mathrm{b}}\right)\right. \\
& \left.\left.+\Lambda_{\mathrm{bp}}\left\{J_{0}^{\prime}\left(\chi_{ \pm, \mathrm{I}, \mathrm{bp}} R_{\mathrm{b}}\right)\left\{-\frac{2 \Omega_{\mathrm{ce}}^{2} \gamma_{0 \mathrm{e}}}{\omega \Delta \omega}+2\right\}\right\}\right\}\right\} \\
& \Theta 1=-J_{0}^{\prime}\left(\chi_{ \pm, \mathrm{II}, \mathrm{p}} R_{\mathrm{b}}\right)-R_{p} J_{-1}\left(\chi_{ \pm, \mathrm{II}, \mathrm{p}} R_{\mathrm{b}}\right), \\
& \Theta 2=Y_{0}^{\prime}\left(\chi_{ \pm, \mathrm{II}, \mathrm{p}} R_{\mathrm{b}}\right)-R_{\mathrm{p}} Y_{-1}\left(\chi_{ \pm, \mathrm{II}, \mathrm{p}} R_{\mathrm{b}}\right), \\
& \Theta 3=\frac{k c R_{\mathrm{p}}}{\omega} J_{-1}\left(\chi_{ \pm, \mathrm{II}, \mathrm{p}} R_{\mathrm{b}}\right), \quad \Theta 4=\frac{k c R_{\mathrm{p}}}{\omega} Y_{-1}\left(\chi_{ \pm, \mathrm{II}, \mathrm{p}} R_{\mathrm{b}}\right), \\
& \Theta 5=\frac{\omega}{2 c k}\left[\left(\varepsilon_{+\mathrm{p}}+2 R_{\mathrm{p}} \varepsilon_{+\mathrm{p}}-1\right) J_{-1}\left(\chi_{ \pm, \mathrm{II}, \mathrm{p}} R_{\mathrm{b}}\right)\right. \\
& \left.+\left(\varepsilon_{-\mathrm{p}}-1\right) J_{1}\left(\chi_{ \pm, \text {II }, \mathrm{p}} R_{\mathrm{b}}\right)\right] \text {, } \\
& \Theta 6=\frac{\omega}{2 c k}\left[\left(\varepsilon_{+\mathrm{p}}+2 R_{\mathrm{p}} \varepsilon_{+\mathrm{p}}-1\right) Y_{-1}\left(\chi_{ \pm, \mathrm{II}, \mathrm{p}} R_{\mathrm{b}}\right)\right. \\
& \left.+\left(\varepsilon_{-\mathrm{p}}-1\right) Y_{1}\left(\chi_{ \pm, \mathrm{II}, \mathrm{p}} R_{\mathrm{b}}\right)\right] \text {, } \\
& \Theta 7=J_{0}^{\prime}\left(\chi_{ \pm, \mathrm{II}, \mathrm{p}} R_{\mathrm{b}}\right)+\frac{1}{2}\left[\left(\varepsilon_{+\mathrm{p}}+2 R_{\mathrm{p}} \varepsilon_{+\mathrm{p}}-1\right) J_{-1}\left(\chi_{ \pm, \mathrm{II}, \mathrm{p}} R_{\mathrm{b}}\right)\right. \\
& \left.-\left(\varepsilon_{-\mathrm{p}}-1\right) J_{1}\left(\chi_{ \pm, \mathrm{II}, p} R_{\mathrm{b}}\right)\right] \text {, } \\
& \Theta 8=Y_{0}^{\prime}\left(\chi_{ \pm, \mathrm{II}, \mathrm{p}} R_{\mathrm{b}}\right)+\frac{1}{2}\left[\left(\varepsilon_{+\mathrm{p}}+2 R_{\mathrm{p}} \varepsilon_{+\mathrm{p}}-1\right)\right. \\
& \left.Y_{-1}\left(\chi_{ \pm, \mathrm{II}, \mathrm{p}} R_{\mathrm{b}}\right)-\left(\varepsilon_{-\mathrm{p}}-1\right) Y_{1}\left(\chi_{ \pm, \mathrm{II}, \mathrm{p}} R_{\mathrm{b}}\right)\right] \text {, }
\end{aligned}
$$

$$
\begin{aligned}
& S= J_{0}^{\prime}\left(\chi_{ \pm, \mathrm{I}, \mathrm{bp}} R_{\mathrm{b}}\right)+\frac{\omega}{2 c k} \frac{\left(c k-\omega \beta_{0 \mathrm{e}}\right)}{\Delta \omega} \\
& \times\left[\varepsilon_{+\mathrm{b}}+\frac{2 \alpha_{\mathrm{b}} \Lambda_{0 \mathrm{~b}}}{\Lambda_{+\mathrm{bp}} \Lambda_{-\mathrm{bp}}} \varepsilon_{+\mathrm{b}}-1\right] J_{-1}\left(\chi_{ \pm, \mathrm{I}, \mathrm{bp}} R_{\mathrm{b}}\right) \\
&- \frac{1}{2}\left(\varepsilon_{-\mathrm{b}}-1\right) J_{1}\left(\chi_{ \pm, \mathrm{I}, \mathrm{bp}} R_{\mathrm{b}}\right)+\frac{1}{2}\left[\varepsilon_{+\mathrm{p}}+\frac{2 \alpha_{\mathrm{p}} \Lambda_{0 \mathrm{p}}}{\Lambda_{+\mathrm{bp}} \Lambda_{-\mathrm{bp}}} \varepsilon_{+p}-1\right] \\
& J_{-1}\left(\chi_{ \pm, \mathrm{I}, \mathrm{bp}} R_{\mathrm{b}}\right)-\left(\varepsilon_{-\mathrm{p}}-1\right) J_{1}\left(\chi_{ \pm, \mathrm{I}, \mathrm{bp}} R_{\mathrm{b}}\right)+F F 1, \\
& T=\frac{\omega}{2 c k}\left[\left(\varepsilon_{+\mathrm{b}}+\frac{2 \alpha_{\mathrm{b}} \Lambda_{0 b}}{\Lambda_{+\mathrm{bp}} \Lambda_{-\mathrm{bp}}} \varepsilon_{+b}-1\right) J_{-1}\left(\chi_{ \pm, \mathrm{I}, \mathrm{bp}} R_{\mathrm{b}}\right)+\left(\varepsilon_{-\mathrm{b}}-1\right)\right. \\
&\left.J_{1}\left(\chi_{ \pm, \mathrm{I}, \mathrm{bp}} R_{\mathrm{b}}\right)\right]+\frac{\omega}{2 c k}\left[\left(\varepsilon_{+\mathrm{p}}+\frac{2 \alpha_{\mathrm{p}} \Lambda_{0 \mathrm{p}}}{\Lambda_{+\mathrm{bp}} \Lambda_{-\mathrm{bp}}} \varepsilon_{+\mathrm{p}}-1\right) J_{-1}\left(\chi_{ \pm, \mathrm{I}, \mathrm{bp}} R_{\mathrm{b}}\right)\right. \\
&\left.+\left(\varepsilon_{-\mathrm{p}}-1\right) J_{1}\left(\chi_{ \pm, \mathrm{I}, \mathrm{bp}} R_{\mathrm{b}}\right)\right]+F F 2,
\end{aligned}
$$$$
F F 1=\frac{\omega}{c k} \frac{1}{\Lambda_{+\mathrm{bp}} \Lambda_{-\mathrm{bp}}}
$$$$
\times\left\{-\frac{\omega_{b}^{2}}{\omega^{2}} \frac{\Delta \omega \alpha_{\mathrm{p}} \Lambda_{0 \mathrm{p}}}{\left(\Delta \omega-\Omega_{\mathrm{ce}}\right)} \frac{c k}{\omega} \frac{\omega_{p}^{2} \gamma_{0 \mathrm{e}}}{\omega} \frac{\alpha_{b} \Lambda_{0 \mathrm{~b}}}{\left(\omega-\gamma_{0 \mathrm{e}} \Omega_{\mathrm{ce}}\right)} \frac{\left(c k-\omega \beta_{0 \mathrm{e}}\right)}{\Delta \omega}\right\}
$$$$
J_{-1}\left(\chi_{ \pm, \mathrm{I}, \mathrm{bp}} R_{\mathrm{b}}\right)+\frac{\omega}{c k}\left(\varepsilon_{r r, I} R 1+i \varepsilon_{r \theta, I} R 2\right),
$$$$
F F 2=\frac{\omega}{c k} \frac{1}{\Lambda_{+\mathrm{bp}} \Lambda_{-\mathrm{bp}}}
$$$$
\times\left\{-\frac{\omega_{b}^{2}}{\omega^{2}} \frac{\Delta \omega \alpha_{\mathrm{p}} \Lambda_{0 \mathrm{p}}}{\left(\Delta \omega-\Omega_{\mathrm{ce}}\right)} \frac{\omega_{p}^{2} \gamma_{0 \mathrm{e}} \alpha_{\mathrm{b}} \Lambda_{0 \mathrm{~b}}}{\omega\left(\omega-\gamma_{0 \mathrm{e}} \Omega_{\mathrm{ce}}\right)}\right.
$$$$
\left.\times J_{-1}\left(\chi_{ \pm, \mathrm{I}, \mathrm{bp}} R_{\mathrm{b}}\right)+\frac{\omega}{c k}\left(\varepsilon_{r r, \mathrm{I}} R 3+i \varepsilon_{r \theta, \mathrm{I}} R 4\right),\right\}
$$$$
R 1=\frac{1}{\Lambda_{+\mathrm{bp}} \Lambda_{-\mathrm{bp}}}\left\{J_{0}^{\prime}\left(\chi_{ \pm, \mathrm{I}, \mathrm{bp}} R_{\mathrm{b}}\right) A_{\mathrm{bp}}\right.
$$$$
R 2=\frac{1}{\Lambda_{+\mathrm{bp}} \Lambda_{-\mathrm{bp}}}\left\{-i J_{0}^{\prime}\left(\chi_{ \pm, \mathrm{I}, \mathrm{bp}} R_{\mathrm{b}}\right) D_{\mathrm{bp}}-\alpha_{\mathrm{b}} \alpha_{\mathrm{p}} J_{0}^{\prime}\left(\chi_{ \pm, \mathrm{I}, \mathrm{bp}} R_{\mathrm{b}}\right)\right.
$$$$
\left\{\left(c k-\omega \beta_{0 \mathrm{e}}\right)\left[\frac{1}{\Delta \omega^{2}}+\frac{\gamma_{0 \mathrm{e}} \Omega_{\mathrm{ce}}}{\omega \Delta \omega^{2}}\right]+c k\left[\frac{1}{\omega^{2}}+\frac{\Omega_{\mathrm{ce}}}{\omega^{2} \Delta \omega}\right]\right\},
$$$$
R 3=\frac{1}{\Lambda_{+\mathrm{bp}} \Lambda_{-\mathrm{bp}}}\left\{-\alpha_{\mathrm{b}} \alpha_{\mathrm{p}} J_{0}^{\prime}\left(\chi_{ \pm, \mathrm{I}, \mathrm{bp}} R_{\mathrm{b}}\right)\right.
$$$$
\left.\times\left[\frac{1}{\Delta \omega}+\frac{\gamma_{0 \mathrm{e}} \Omega_{\mathrm{ce}}}{\omega \Delta \omega}+\frac{1}{\omega}+\frac{\Omega_{\mathrm{ce}}}{\omega \Delta \omega}\right]\right\},
$$$$
R 4=\frac{1}{\Lambda_{+\mathrm{bp}} \Lambda_{-\mathrm{bp}}}\left\{-B_{\mathrm{bp}} J_{0}^{\prime}\left(\chi_{ \pm, \mathrm{I}, \mathrm{bp}} R_{\mathrm{b}}\right)\right\}
$$$$
\varepsilon_{ \pm \mathrm{b}}=1-\frac{\omega_{\mathrm{b}}^{2}}{\omega^{2}} \frac{\Delta \omega_{\mathrm{m}}}{\left(\Delta \omega_{\mathrm{m}} \mp \Omega_{\mathrm{ce}}\right)}, \varepsilon_{ \pm \mathrm{p}}=1-\frac{\omega_{\mathrm{p}}^{2} \gamma_{0 \mathrm{e}}}{\omega\left(\omega \mp \Omega_{\mathrm{ce}} \gamma_{0 \mathrm{e}}\right)},
$$

By imposing the following conditions at the helix

$$
\begin{aligned}
& \delta \hat{E}_{z}\left(R_{\mathrm{h}}-\varepsilon\right)=\delta \hat{E}_{z}\left(R_{\mathrm{h}}+\varepsilon\right) \\
& \delta \hat{E}_{\theta}\left(R_{\mathrm{h}}-\varepsilon\right)=\delta \hat{E}_{\theta}\left(R_{\mathrm{h}}+\varepsilon\right)
\end{aligned}
$$

$D$ and $K$ are given by 


$$
\begin{aligned}
D= & \frac{1}{W\left(q R_{\mathrm{g}}, q R_{\mathrm{h}}\right)}\left\{A\left[a^{(1)} J_{0}\left(\chi_{ \pm, \mathrm{II}, \mathrm{p}} R_{\mathrm{h}}\right)+a^{(2)} Y_{0}\left(\chi_{ \pm, \mathrm{II}, \mathrm{p}} R_{\mathrm{h}}\right)\right]\right. \\
& \left.+i F\left[c^{(1)} J_{0}\left(\chi_{ \pm, \mathrm{II}, \mathrm{p}} R_{\mathrm{h}}\right)+c^{(2)} Y_{0}\left(\chi_{ \pm, \mathrm{II}, \mathrm{p}} R_{\mathrm{h}}\right)\right]\right\} \\
K= & \frac{q \chi_{ \pm, \mathrm{II}, \mathrm{p}}}{\chi^{2} W^{\prime \prime}\left(q R_{\mathrm{g}}, q R_{\mathrm{h}}\right)}\left[F \phi^{(1)}+i A \phi^{(2)}\right],
\end{aligned}
$$

where

$$
\begin{aligned}
\phi^{(1)} & =\left[b^{(1)} J_{0}^{\prime}\left(\chi_{ \pm, I I, p} R_{h}\right)+b^{(2)} Y_{0}^{\prime}\left(\chi_{ \pm, \mathrm{II}, \mathrm{p}} R_{\mathrm{h}}\right)\right] \\
& +\frac{c k R_{\mathrm{p}}}{\omega}\left[c^{(1)} J_{0}^{\prime}\left(\chi_{ \pm, \mathrm{II}, \mathrm{p}} R_{\mathrm{h}}\right)+c^{(2)} Y_{0}^{\prime}\left(\chi_{ \pm, \mathrm{II}, \mathrm{p}} R_{\mathrm{h}}\right)\right] \\
& +R_{\mathrm{p}}\left[b^{(1)} J_{0}^{\prime}\left(\chi_{ \pm, \mathrm{II}, \mathrm{p}} R_{\mathrm{h}}\right)+b^{(2)} Y_{0}^{\prime}\left(\chi_{ \pm, \mathrm{II}, \mathrm{p}} R_{\mathrm{h}}\right)\right] \\
\phi_{1, \mathrm{~m}}^{(2)} & =\left[d^{(1)} J_{0}^{\prime}\left(\chi_{ \pm, \mathrm{II}, \mathrm{p}} R_{\mathrm{h}}\right)+d^{(2)} Y_{0}^{\prime}\left(\chi_{ \pm, \mathrm{II}, \mathrm{p}} R_{\mathrm{h}}\right)\right] \\
& -\frac{c k R_{\mathrm{p}}}{\omega}\left[a^{(1)} J_{0}^{\prime}\left(\chi_{ \pm, \mathrm{II}, \mathrm{p}} R_{\mathrm{h}}\right)+a^{(2)} Y_{0}^{\prime}\left(\chi_{ \pm, \mathrm{II}, \mathrm{p}} R_{\mathrm{h}}\right)\right] \\
& +R_{\mathrm{p}}\left[d^{(1)} J_{0}^{\prime}\left(\chi_{ \pm, \mathrm{II}, \mathrm{p}} R_{\mathrm{h}}\right)+d^{(2)} Y_{0}^{\prime}\left(\chi_{ \pm, \mathrm{II}, \mathrm{p}} R_{\mathrm{h}}\right)\right]
\end{aligned}
$$

To obtain the final dispersion relation we must employ the discontinuity conditions in the axial and azimuthal magnetic fields due to the helix current sheet as follows

$$
\begin{aligned}
& \delta B_{\theta}\left(R_{\mathrm{h}}+\varepsilon\right)-\delta B_{\theta}\left(R_{\mathrm{h}}-\varepsilon\right) \\
& \quad=\frac{4 \pi}{c} \delta J_{11} \Delta R_{\mathrm{h}} \sin \varphi \text { and } \delta \hat{B}_{z}\left(R_{\mathrm{h}}-\varepsilon\right)-\delta \hat{B}_{z}\left(R_{\mathrm{h}}+\varepsilon\right) \\
& \quad=\frac{4 \pi}{c} \delta \hat{J}_{11} \Delta R_{\mathrm{h}} \cos \varphi,
\end{aligned}
$$

By employing Eq. 8 and onerous manipulations, we get:

$$
\begin{aligned}
A= & {\left[-i W^{\prime \prime}\left(q R_{\mathrm{g}}, q R_{\mathrm{h}}\right) \cos \varphi T_{3}-i \chi^{2} \sin \varphi T_{1}\right] /\left[c \left(T_{1} T_{4}\right.\right.} \\
& \left.\left.+T_{3} T_{2}\right)\right], \\
F= & 4 \pi \delta J_{11} \Delta R_{\mathrm{h}}\left[W^{\prime \prime}\left(q R_{\mathrm{g}}, q R_{\mathrm{h}}\right) \cos \varphi T_{4}-\chi^{2} \sin \varphi T_{2}\right] \\
& \times\left[c\left(T_{1} T_{4}+T_{3} T_{2}\right)\right],
\end{aligned}
$$

where $T_{1}, T_{2}, T_{3}, T_{4}$ are as follows:

$$
\begin{aligned}
T_{1}= & {\left[b^{(1)} J_{0}\left(\chi_{ \pm, \mathrm{II}, \mathrm{p}} R_{\mathrm{h}}\right)+b^{(2)} Y_{0}\left(\chi_{ \pm, \mathrm{II}, \mathrm{p}} R_{\mathrm{h}}\right)\right] W^{\prime \prime}\left(q R_{\mathrm{g}}, q R_{\mathrm{h}}\right) } \\
& -\frac{q \chi_{ \pm, \mathrm{II}, \mathrm{p}}}{\chi^{2}} W^{\prime}\left(q R_{\mathrm{g}}, q R_{\mathrm{h}}\right) \phi^{(1)} \\
T_{2}= & {\left[d^{(1)} J_{0}\left(\chi_{ \pm, \mathrm{II}, \mathrm{p}} R_{\mathrm{h}}\right)+b^{(2)} Y_{0}\left(\chi_{ \pm, \mathrm{II}, \mathrm{p}} R_{\mathrm{h}}\right)\right] } \\
& \times W^{\prime \prime}\left(q R_{\mathrm{g}}, q R_{\mathrm{h}}\right)-\frac{q \chi_{ \pm, \mathrm{II}} \mathrm{p}}{\chi^{2}} W^{\prime}\left(q R_{\mathrm{g}}, q R_{\mathrm{h}}\right) \phi^{(2)} \\
T_{3}= & +\frac{\chi^{2} \varepsilon \omega}{c q W\left(q R_{\mathrm{g}}, q R_{\mathrm{h}}\right)}\left[Y_{0}\left(q R_{\mathrm{g}}\right) J_{0}^{\prime}\left(q R_{\mathrm{h}}\right)-J_{0}\left(q R_{\mathrm{g}}\right)\right. \\
& \left.Y_{0}^{\prime}\left(q R_{\mathrm{h}}\right)\right]\left[c^{(1)} J_{0}\left(\chi_{ \pm, \mathrm{II}, \mathrm{p}} R_{\mathrm{h}}\right)+c^{(2)} Y_{0}\left(\chi_{ \pm, \mathrm{II}, \mathrm{p}} R_{\mathrm{h}}\right)\right] \\
& -\left[\frac{\chi_{ \pm, \mathrm{II}, \mathrm{p}}}{c}+k R_{\mathrm{p}} \frac{c k}{\omega} \chi_{ \pm, \mathrm{II}, \mathrm{p}}\right]\left[c^{(1)} J_{0}^{\prime}\left(\chi_{ \pm, \mathrm{II}, \mathrm{p}} R_{\mathrm{h}}\right)+c^{(2)} Y_{0}^{\prime}\left(\chi_{ \pm, \mathrm{II}, \mathrm{p}} R_{\mathrm{h}}\right)\right] \\
& -k R_{\mathrm{p}} \chi_{ \pm, \mathrm{II}, \mathrm{p}}\left[b^{(1)} J_{0}^{\prime}\left(\chi_{ \pm, \mathrm{II}, \mathrm{p}} R_{\mathrm{h}}\right)+b^{(2)} Y_{0}^{\prime}\left(\chi_{ \pm, \mathrm{II}, \mathrm{p}} R_{\mathrm{h}}\right)\right] \\
T_{4}= & \frac{\chi^{2} \varepsilon \omega}{c q W\left(q R_{g}, q R_{h}\right)}\left[Y_{0}\left(q R_{\mathrm{g}}\right) J_{0}^{\prime}\left(q R_{\mathrm{h}}\right)\right. \\
& \left.-J_{0}\left(q R_{\mathrm{g}}\right) Y_{0}^{\prime}\left(q R_{\mathrm{h}}\right)\right]\left[a^{(1)} J_{0}\left(\chi_{ \pm, \mathrm{II}, \mathrm{p}} R_{\mathrm{h}}\right)+a^{(2)} Y_{0}\left(\chi_{ \pm, \mathrm{II}, \mathrm{p}} R_{\mathrm{h}}\right)\right] \\
& -\left[\frac{\chi_{ \pm, \mathrm{II}, \mathrm{p}} \omega}{c}+k R_{\mathrm{p}} \frac{c k}{\omega} \chi_{ \pm, \mathrm{II}, \mathrm{p}}\right]\left[a^{(1)} J_{0}^{\prime}\left(\chi_{ \pm, \mathrm{II}, \mathrm{p}} R_{\mathrm{h}}\right)+a^{(2)} Y_{0}^{\prime}\left(\chi_{ \pm, \mathrm{II}, \mathrm{p}} R_{\mathrm{h}}\right)\right] \\
& +k R_{\mathrm{p}} \chi_{ \pm, \mathrm{II}, \mathrm{p}}\left[d^{(1)} J_{0}^{\prime}\left(\chi_{ \pm, \mathrm{II}, \mathrm{p}} R_{\mathrm{h}}\right)+d^{(2)} Y^{\prime}\left(\chi_{ \pm, \mathrm{II}, \mathrm{p}} R_{\mathrm{h}}\right)\right]
\end{aligned}
$$

The final condition says that the electric field parallel to the helix must be zero [18],

$\left[\delta E_{z} \sin \varphi+\delta E_{z} \cos \varphi\right]=0$,

The dispersion relation obtained by employing Eqs. (9)-(11).

$$
\left\{\Psi^{(1)}+\Psi^{(2)}\right\}=0 \text {, }
$$

where

$$
\begin{aligned}
& \Psi^{(1)}=\frac{4 \pi \delta \hat{J}_{\|} \Delta R_{h}}{c}\left[\left[-i W^{\prime \prime}\left(\mathrm{g}, \chi R_{\mathrm{h}}\right) \cos \varphi T_{3}-i \chi^{2} \sin \varphi T_{1}\right] /\right. \\
& \left.\left(T_{1} T_{4}+T_{3} T_{2}\right)\right]\left[\left[a^{(1)} J_{0}\left(\chi_{ \pm, \mathrm{II}, \mathrm{p}} R_{\mathrm{h}}\right)+a^{(2)} Y_{0}\left(\chi_{ \pm, \mathrm{II}, \mathrm{p}} R_{\mathrm{h}}\right)\right]\right. \\
& \sin \varphi+\left[d^{(1)} J_{0}^{\prime}\left(\chi_{ \pm, \mathrm{II}, \mathrm{p}} R_{\mathrm{h}}\right)+d^{(2)} Y_{0}^{\prime}\left(\chi_{ \pm, \mathrm{II}, \mathrm{p}} R_{\mathrm{h}}\right)\right] \\
& \times\left(\frac{\omega \chi_{ \pm, \mathrm{II}, \mathrm{p}}}{c \chi^{2}} \cos \varphi\left[1+R_{\mathrm{p}}(\omega, k)\right]\right) \\
& \left.-\frac{k \chi_{ \pm, \mathrm{II}, \mathrm{p}}}{\chi^{2}} R_{\mathrm{p}}(\omega, k) \cos \varphi\left[a^{(1)} J_{0}^{\prime}\left(\chi_{ \pm, \mathrm{II}, \mathrm{p}} R_{\mathrm{h}}\right)+a^{(2)} Y_{0}^{\prime}\left(\chi_{ \pm, \mathrm{II}, \mathrm{p}} R_{\mathrm{h}}\right)\right]\right],
\end{aligned}
$$

and

$$
\begin{gathered}
\psi^{(2)}=\frac{i 4 \pi \delta \hat{J}_{\|} \Delta R_{\mathrm{h}}}{c}\left[\left[W^{\prime \prime}\left(\chi R_{\mathrm{g}}, \chi R_{\mathrm{h}}\right) \cos \varphi T_{4}-\chi^{2} \sin \varphi T_{2}\right]\right. \\
\left./\left(T_{1} T_{4}+T_{3} T_{2}\right)\right]\left[\left[c^{(1)} J_{0}\left(\chi_{ \pm, \mathrm{II}, \mathrm{p}} R_{\mathrm{h}}\right) c^{(2)} Y_{0}\left(\chi_{ \pm, \mathrm{II}, \mathrm{p}} R_{\mathrm{h}}\right)\right]\right. \\
\sin \varphi-\left[b^{(1)} J_{0}^{\prime}\left(\chi_{ \pm, \mathrm{II}, \mathrm{p}} R_{\mathrm{h}}\right)+b^{(2)} Y_{0}^{\prime}\left(\chi_{ \pm, \mathrm{II}, \mathrm{p}} R_{\mathrm{h}}\right)\right] \\
\times\left(\frac{\omega \chi_{ \pm, \mathrm{II}, \mathrm{p}}}{c^{2}} \cos \varphi\left[1+R_{\mathrm{p}}\right]\right)-\frac{k \chi_{ \pm, \mathrm{II}, \mathrm{p}}}{\chi^{2}}
\end{gathered}
$$$$
\left.R_{\mathrm{p}} \cos \varphi\left[c^{(1)} J_{0}^{\prime}\left(\chi_{ \pm, \mathrm{II}, \mathrm{p}} R_{\mathrm{h}}\right)+c^{(2)} Y_{0}^{\prime}\left(\chi_{ \pm, \mathrm{II}, \mathrm{p}} R_{\mathrm{h}}\right)\right]\right],
$$

\section{Numerical results and discussion}

We analyze the dispersion characteristics of the slow-wave structure from the numerical computation of the dispersion using (12). The nominal parameters of this system correspond to a helix with a period $\lambda_{\mathrm{h}}=1.966 \mathrm{~cm}$ a width $\xi_{\mathrm{h}}=0.764 \mathrm{~cm}$, and a radius of $R_{\mathrm{h}}=1.4 \mathrm{~cm}$ enclosed within a wall of radius $R_{\mathrm{w}}=3.63 \mathrm{~cm}$. Consider only the case in which $m=0$. The growth rate is proportional to the Pierce gain parameter known in the theory of TWTs $C=\left(Z I_{\mathrm{b}} /\left(4 V_{\mathrm{b}}\right)\right)^{1 / 3}$. Where $I_{\mathrm{b}}$ is the electron beam current and $Z$ is the coupling impedance of electrons to the wave, which depends on the transverse structure of the wave in the beam region. The coupling impedance of solid electron beam in the presence of plasma can be much larger than the vacuum case [22].

First, we consider the plasma density is weak. Figure 2 shows the variation of the plasma velocity as a function of the frequency for several values of plasma frequency. As seen in this figure, the plasma velocity decreases with frequency, and it remains constant with plasma density. 


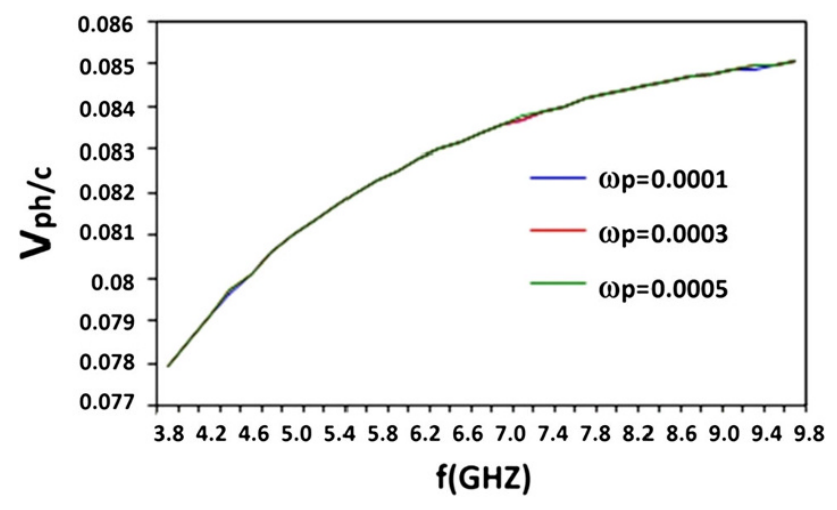

Fig. 2 Variation of the normalized phase velocity $\left(v_{\mathrm{ph}} / c\right)$ with frequency $(f)$ for several values of the plasma density (low plasma density). The chosen parameters are as follows: $\omega_{\mathrm{b}}=0.025$, $\Omega_{\mathrm{ce}}=0.07$, and $\varepsilon=1.75$

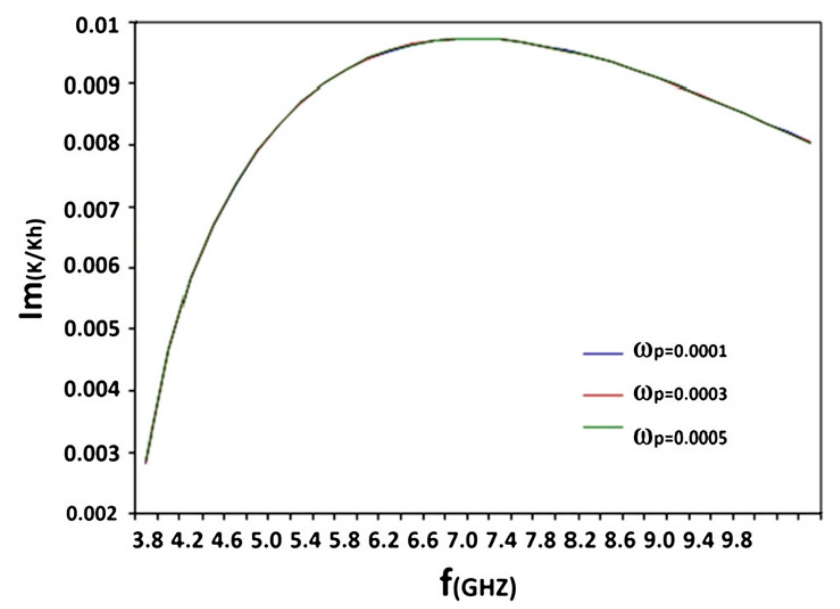

Fig. 3 Variation of the normalized growth rate $\left(I_{m} k / k_{\mathrm{h}}\right)$ with frequency $(f)$ for several values of the plasma density (low plasma density). The chosen parameters are as follows: $\omega_{\mathrm{b}}=0.025$, $\Omega_{\mathrm{ce}}=0.07, \gamma_{0 \mathrm{e}}=1.005558, \varepsilon=1.75$

The plot of gain as a function of frequency for different values of plasma density is shown in Fig. 3. In accordance with Fig. 2, the growth rate is constant by increasing plasma density. This shows that the coupling impedance in the lower plasma density is weak and growth rate remains constant in this limit.

Now, we consider the numerical solution for strong plasma density. Figure 4 shows the phase velocity as a function of frequency for several choice of plasma density. It is clear from Fig. 4 that the phase velocity increases with frequency, and the plasma density increases the phase velocity.

Figure 5 illustrates the variation of gain as a function of frequency for different values of plasma density. Figure 5 has a good agreement with Fig. 4. As seen in this figure, the effect of plasma is to enhance the gain in the strong

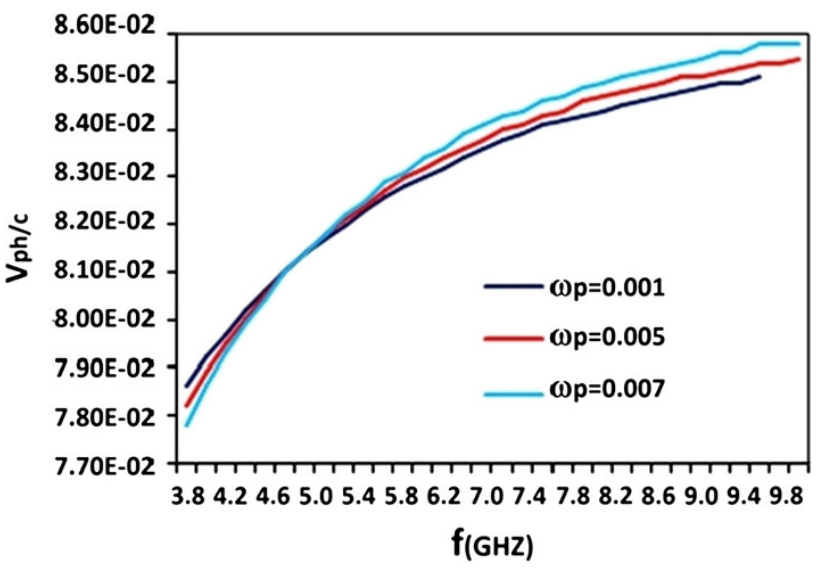

Fig. 4 Variation of the normalized phase velocity $\left(v_{\mathrm{ph}} / c\right)$ with frequency $(f)$ for several values of the plasma density (high plasma density). The chosen parameters are as follows: $\omega_{\mathrm{b}}=0.025$, $\Omega_{\mathrm{ce}}=0.07$, and $\varepsilon=1.75$

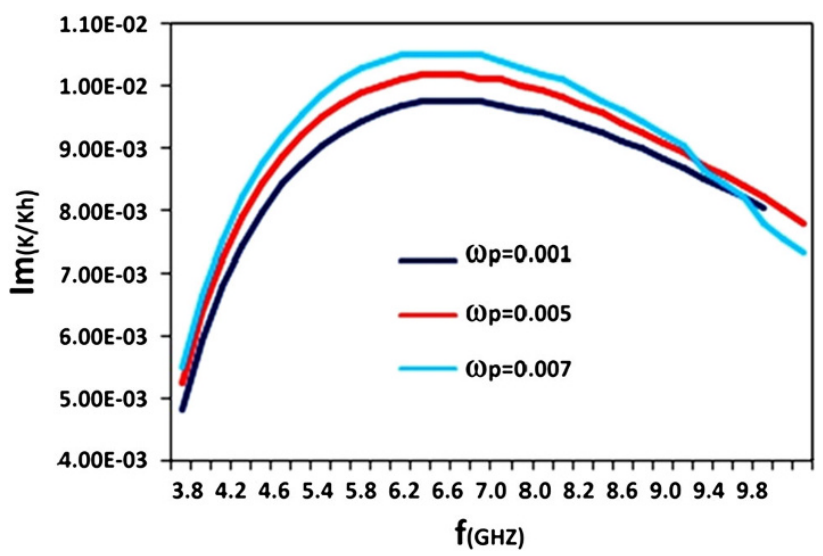

Fig. 5 Variation of the normalized growth rate $\left(I_{\mathrm{m}} k / k_{\mathrm{h}}\right)$ with frequency $(f)$ for several values of the plasma density (high plasma density). The chosen parameters are as follows: $\omega_{\mathrm{b}}=0.025$, $\Omega_{\mathrm{ce}}=0.07, \gamma_{0 \mathrm{e}}=1.005558, \mathrm{R} \varepsilon=1.75$

plasma density limit. The presence of plasma should increase the growth rate of the electromagnetic wave. The presence of strong plasma is to increase the coupling impedance.

In the absence of the plasma the dispersion relation reduce to the final dispersion relation in the Ref. [18, 19]. The comparison of the growth rate in the absence and presence of the plasma is shown in Fig. 6. As seen in this figure, the effect of plasma is to increase the growth rate and frequency bandwidth.

\section{Conclusion}

Now, we can summarize the specific results of this project as: 


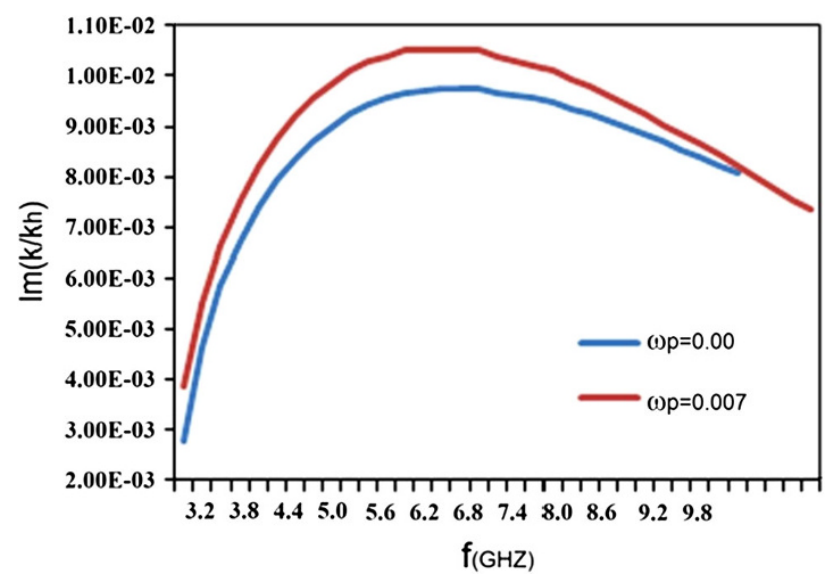

Fig. 6 The comparison of the growth rate as a function of frequency in the presence and absence of the plasma. The chosen parameters are as: $\omega_{\mathrm{b}}=0.025, \Omega_{\mathrm{ce}}=0.07, \gamma_{0 \mathrm{e}}=1.005558, \varepsilon=1.75$

1. In the lower plasma density, the effect of plasma on the growth rate and frequency bandwidth is not considerable.

2. The effect of strong plasma is to increase the growth rate and frequency bandwidth.

3. The coupling impedance is strong in the strong plasma density.

4. Presence of plasma in the system considerably increases the growth rate and bandwidth in comparison with the absence of the plasma.

Open Access This article is distributed under the terms of the Creative Commons Attribution License which permits any use, distribution, and reproduction in any medium, provided the original author(s) and the source are credited.

\section{References}

1. Shiffler, D., Nation, J.A., Graham, S.K.: A high-power, traveling wave tube amplifier. IEEE Trans. Plasma Sci. 18, 546 (1990)

2. Sawhney, R., Maheshwari, K.P., Choyal, Y.: Effect of plasma on efficiency enhancement in a high power relativistic backward wave oscillator. IEEE Trans. Plasma Sci. 21, 609 (1993)

3. Zavjalov, M.A., Mitin, L.A., Perevodchikov, V.I., Tskhai, V.N., Shapiro, A.L.: Powerful wideband amplifier based on hybrid plasma-cavity slow-wave structure. IEEE Trans. Plasma Sci. 22, 600 (1994)

4. Nusinovich, G.S., Carmel Jr, Y., Antonsen, T.M.: Recent progress in the development of plasma-filled traveling-wave tubes and backward-wave oscillators. IEEE Trans. Plasma Sci. 26, 628 (1998)

5. Kobayashi Jr, S., Antonsen, T.M., Nusinovich, G.S.: Linear theory of a plasma loaded, helix type, slow wave amplifier. IEEE Trans. Plasma Sci. 26, 669 (1998)

6. Pierce, J.R.: Travelling-Wave Tubes. Van Nostrand Reinhold, ch. 3, New York (1950)

7. Hutter, R.G.: Beam and Wave Electronics in Microwave Tubes. Van Nostrand, ch. 7, New York (1960)

8. Watkins, D.A.: Topics in Electromagnetic Theory. Wiley, ch. 3, New York (1958)

9. Uhm, H.S., Choe, J.Y.: Properties of the electromagnetic wave propagation in a helix loaded waveguide. J. Appl. Phys. 53, 8483 (1982)

10. XiaoFang, Z., ZhongHai, Y., Bin, L.: Modeling of T-shaped vane loaded helical slow-wave structures. IEEE Trans. Plasma Sci. 34, 563-571 (2006)

11. Pierce, J.R., Field, L.M.: Traveling-wave tubes. Proc. IRE 35, 108 (1947)

12. Chu, L.J., Jackson, J.D.: Field theory of traveling-wave tubes. Proc. IRE 36, 853-863 (1945)

13. Wei, Y.-Y., Wang, W.-X., Sun, J.-H., Liu, S.-G., Bao fu, J., Park, G.-S.: Dielectric effect on the $\mathrm{rf}$ characteristics of a helical groove travelling wave tube. Chin. Phys. B. 11, 277-281 (2002)

14. Rydbeck, O.E.H.: Theory of the traveling wave tube. Ericsson Technol. 46, 3 (1948)

15. Chodorow, M., Nalos, N.J.: The design of high-power travelingwave tubes. Proc. IRE 44, 649-659 (1956)

16. Beck, A.H.W.: Space-Charge Waves. Pergamon, New York (1958)

17. Hutter, R.G.E.: Beam and Wave Electronics in Microwave Tubes. VanNostrand, New York (1960)

18. Freund, H.P., Vanderplaats, N.R., Kodis, M.A.: Field theory of a traveling wave tube amplifier with a tape helix. IEEE Trans. Plasma Sci. 21, 654-668 (1993)

19. Freund, H.P., Kodis, M.A., Vanderplaats, N.R.: Self-consistent field theory of a helix traveling wave tube amplifier. IEEE Trans. Plasma Sci. 20, 543-553 (1992)

20. Kumar, M., Bhasin, L., Tripathi, V.K.: Plasma effects in a travelling wave tube. Phys. Scr. 81(1-6), 025502 (2010)

21. Pu-Kun, Liu, Hong-Quan, Xie: Theoretical analysis of a relativistic travelling wave tube filled with plasma. Chin. Phys. B. 16, 766-771 (2007)

22. Nusinovich, G.S., Mitin, L.A., Vlasov, A.N.: Space charge effects in plasma-filled traveling-wave tubes. Phys. Plasma 4, 4394 (1997) 